\title{
PREDICTORS OF PATELLOFEMORAL PAIN APPLYING FULL WEIGHT BEARING KINEMATIC MRI
}

\author{
Andrius Brazaitis, , A, B, C, D Algirdas Tamošiūnas, ${ }^{1, A, D}$ Janina Tutkuvienè, 2, A, D \\ Audrius Gocentas ${ }^{3, D}$
}

\footnotetext{
${ }^{1}$ Department of Radiology, Vilnius University Faculty of Medicine, Vilnius, Lithuania

2 Department of Anatomy, Histology and Anthropology, Vilnius University Faculty of Medicine, Vilnius, Lithuania

${ }^{3}$ State Research Institute Centre for Innovative Medicine, Vilnius, Lithuania

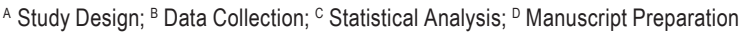

\author{
Address for corpespondence: \\ Andrius Brazaitis, MD \\ Vilnius University Faculty of Medicine, Department of Radiology \\ Santariškiu 2, Vilnius, Lithuania, \\ E-mail: andrius.brazaitis@santa.lt
}

\begin{abstract}
Ahstract. Purpose. To determine the associations among PFM, trochlea groove morphology, femoral and patellar rotation and to determine which measures best predict patellofemoral pain (PFP).

Material \& Methods. Knees of 51 female patients with PFP and 26 healthy female volunteers as control group were analysed with kinematic magnetic resonance imaging and full weight-bearing. The bisect offset (BSO), patellar tilt angle (PTA), femoral rotation angle (FRA), patellar rotation angle (PRA) were measured in steps of $10^{\circ}$ between $0^{\circ}$ to $50^{\circ}$ of knee flexion. Static measures of Insall-Salvati ratio (ISR), sulcus angle (SAB \& SAC) and lateral trochlear inclination at bone and cartilage levels (LTIB \& LTIC) were performed and compared.

Results. The SAB \& SAC were higher and the LTIB \& LTIC were lower in patients compared to volunteers. BSO, PTA, PRA and medial FRA were higher in the PFP group at all flexion angles. A multiple logistic regression analysis demonstrated that increase of FRA and SAB by $1^{\circ}$ increased the likelihood of PFP 5.6 times and 1.6 times respectively, decrease of PRA by $1^{\circ}$ decrease PFP likelihood by 1.7 times.

Conclusion. These results revealed FRA, SAB and PRA to have best predictive value for patellofemoral pain. Current findings can help to assess the complexity of predisposing factors for PFP in practice.
\end{abstract}

Key WOrlls: patellofemoral pain, patellofemoral malalignment, kinematic MRI, femoral rotation

\section{Introduction}

Patellofemoral pain (PFP) is a common knee disorder characterized by a diffuse pain over anterior aspect of the knee and aggravated by squatting, running, jumping or other activities that increase patellofemoral joint compressive forces (Wilson 2007). This multifactorial condition often is diagnosed in physically active people and can be described as chronic problem reducing their athletic ability. Limited effectiveness of treatment is associated 
with a different genesis of PFP. Local, trunk and distal factors that influence PFP are determined and studied (Wilson 2007; Witvrouw et al. 2014). Patellofemoral maltracking was found in $50 \%$ of patients and currently could be named as mostly accepted reason for PFP (Pal et al. 2013).

Patella alta is believed to be one of the possible causes for PFP and instability (Pal et al. 2013). Patients with patella alta are at $20-30 \%$ greater risk for PFP and patellar displacement but the influence of patellar height for patellar instability is still controversial (Ward et al. 2007; Stefanik et al. 2012; Biyani et al. 2014; Teng et al. 2014).

Femoral trochlea sulcus angle is a static factor for patellar stability. Flattened sulcus predispose patella to displace laterally as the quadriceps muscle contracts. Angle between $140-150^{\circ}$ is believed to cause PFP (Insall et al. 1983). It has been reported that there is a mismatch between the bony and cartilaginous anatomy of the trochlea (Staubli et al. 1999; Shih et al. 2004) but possible influence on patellar instability was not evaluated. Harbaugh et al. (2010) and Teng et al. (2014) reported that the lateral trochlear inclination (LTI) was more important in predicting patellar lateral displacement, than sulcus angle.

It is widely accepted, that the unstable patella slides over the stable femur (Wittstein et al. 2006). However altered patellofemoral joint kinematics during weight bearing may be more associated with abnormal femur motion than patella motion. Greater lateral patellar tilt and displacement, greater amount of medial femoral rotation were associated with PFP (Powers 2010; Souza et al. 2010). We hypothesized that females with PFP might have greater medial femoral rotation when compared to the pain free individuals.

Different opinions exist regarding morphometric characteristics of the patellofemoral joint and their influence on patellar alignment and PFP (Powers 2000; Harbaugh et al. 2010; Varadarajan et al. 2010). The purpose of this study was to use full weight bearing kinematic MRI, to systematically examine relationships among patellofemoral malalignment; morphologic features of the trochlea groove, femoral and patellar rotation and to determine which measures are the best predictors for PFP.

\section{Materials and methods}

Fifty one females with PFP and twenty six pain free females aged between 18-40 years were included in the study. Before MRI study all subjects were informed as to the nature of the study and signed a human subject's consent form approved by the Vilnius Regional Biomedical Research Ethics Committee. Inclusion criteria were for at least two symptoms associated with PFP: painful stair ascent or descent, painful squatting, painful kneeling, painful prolonged sitting. Exclusion criteria were: age $>40$ years, previous knee surgery, traumatic patellar dislocation, any implanted pacemakers, cochlear implants, clips. The kinematic MRI examination was done with a $1.5 \mathrm{~T}$ MRI unit (Siemens Symphony, Erlangen, Germany) using body coil to allow flexion and extension of both knees. We used a Dynawell L-spine device to load patients with full weight and MRI machine table movement to let knees extend from $50^{\circ}$ to $0^{\circ}$. Patients in supine position were asked to press upon the Dynawell L-spine device using both legs with equal force. The table was moved automatically to extend knees by $10^{\circ}$. Sagittal and axial images of the patellofemoral joint were acquired with a transmit-receive surface body coil and a fast gradient-echo pulse sequence. Sagittal images were acquired to double check the knee flexion angle. Scanning parameters: repetition time (TR) 2.84 milliseconds, echo time (TE), 1.05 milliseconds; flip angle $8^{\circ}$, field of view $30 \times 30 \mathrm{~cm}$, matrix $256 \times 128$, slice thickness, $3 \mathrm{~mm}$; number of slices 30 , excitations 1 .

Images with maximum patellar width were selected for evaluation of medial femoral rotation, patella rotation, lateral patella tilt and lateral patella displacement. Medial/lateral femoral rotation (FRA) was measured as the 
angle formed by the line joining the posterior femoral condyles and the line parallel to the horizontal orientation of the field of view (Figure 1a). Patella rotation (PRA) was measured as the angle formed by the line defining the maximum patella width and a line parallel to the horizontal orientation of the field of view (Figure 1b). Medial/ lateral patella tilt (PTA) was measured as the angle formed by the line joining the maximum width of the patella and the line joining the posterior femoral condyles (Figure 1c). Positive values for femoral rotation indicate medial rotation, negative - external. As with femoral rotation, medial patella rotation was defined as positive and lateral patella rotation as negative. Medial/lateral patellar displacement (Figure 1d) was measured using the bisect offset (BSO) index as described by Brossman et al. (1993). A line was drawn parallel to posterior femoral condyles, while another perpendicular line was drawn through the deepest trochlear point. This line intersects the line connecting the widest patellar points. Measurements were made with Leonardo workstation (Siemens, Erlangen, Germany). The femoral and patella rotation angle, patella tilt angle and the bisect offset measurements were collected from images acquired with full weight bearing from $50^{\circ}$ to full extension. Femoral rotation, patella rotation and lateral patella tilt measurements were reported in degrees, lateral patella displacement (bisect offset) was expressed in percent.

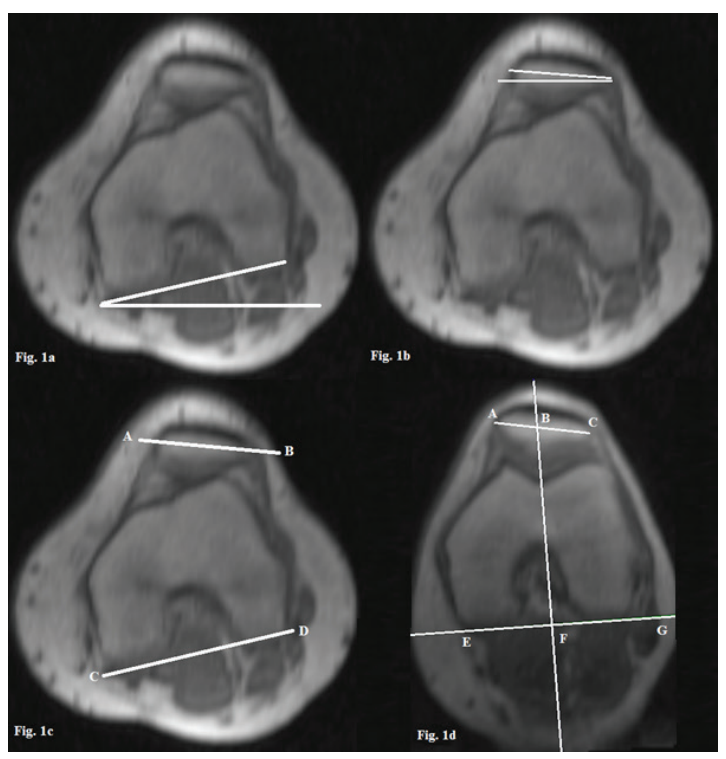

Figure 1. Descriptions of analyzed patellofemoral kinematic parameters. (a) femoral rotation (FRA) - angle between posterior femoral condyles and external field of view; (b) patellar rotation angle (PRA) formed by a line connecting the widest patellar points and external field of view; (c) patella tilt angle (PTA) formed between lines connecting the widest patellar points and posterior femoral condyles; (d) Bisect offset (BSO). EG line parallel to posterior femoral condyles, BF line perpendicular to EG line bisects AC line connecting the widest patellar points $\mathrm{A}$ and $\mathrm{C}$. BSO is $\mathrm{BC} / \mathrm{AC} \times 100$

Insall-Salvati ratio (ISR) was measured from sagittal images. Ratio was calculated by dividing the distance from the tibial tuberosity to the inferior pole of the patella by the length of the patella measured by the distance from the apex of the patella to its most posterior superior point (Figure 2). 


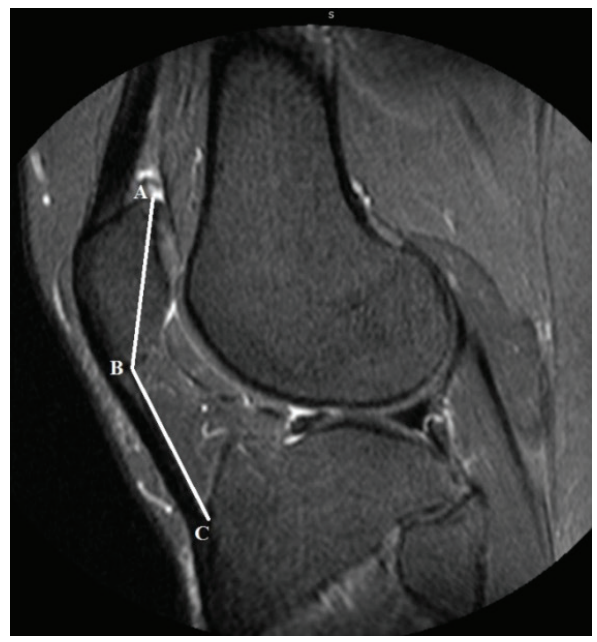

Figure 2. Insall-Salvati ratio was calculated by dividing the length of the patella tendon $(\mathrm{BC})$ by the length of the patella (AB)

Measurements for sulcus angle and lateral trochlear inclination were made at first proximal axial image where cartilage completely covered trochlear groove. Sulcus angle is defined as the angle connecting highest points of the medial and lateral trochlear facets and the deepest point of the trochlear groove. Sulcus angle at bone level (SAB) and sulcus angle at cartilage level (SAC) were measured (Figure 3). Lateral trochlear inclination (LTI) is the angle of the lateral ridge of the trochlear groove with respect to the posterior condyles. LTI was measured at the bone level (LTIB) and cartilage level (LTIC).
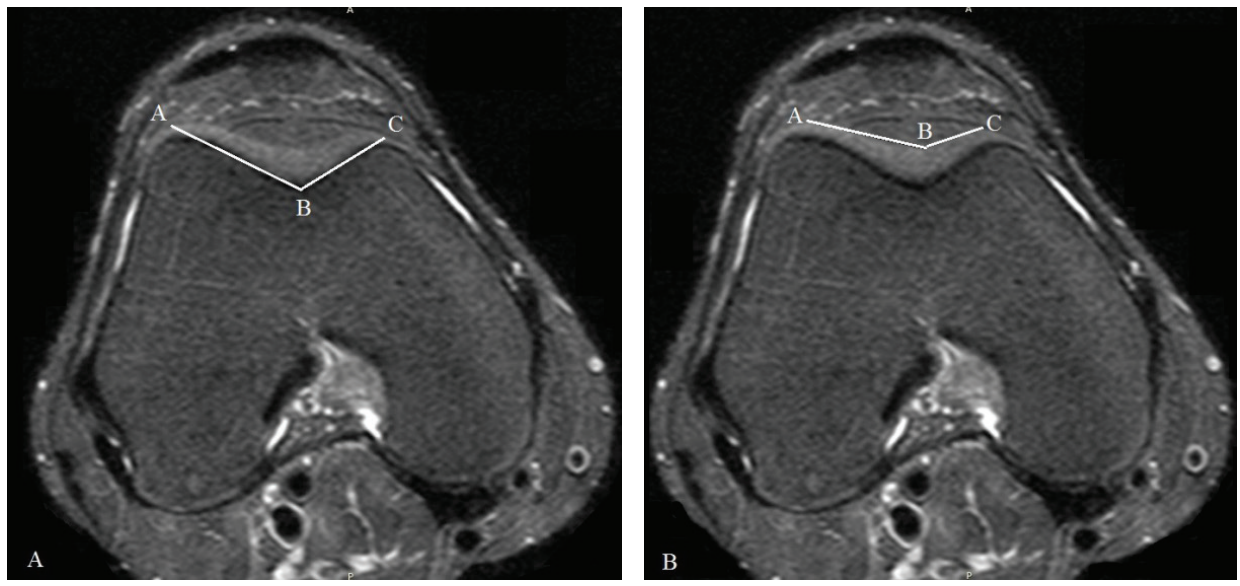

Figure 3. (A) Sulcus angle at the bone level (SAB) is the angle formed by the highest point of the medial and lateral anterior femoral condyles and the deepest point of the femoral trochlear groove at the bone level, (B) sulcus angle at the cartilage level (SAC) is the angle formed by the highest point of the medial and lateral anterior femoral condyles and the deepest point of the femoral trochlear groove at the bone level at the cartilage level 

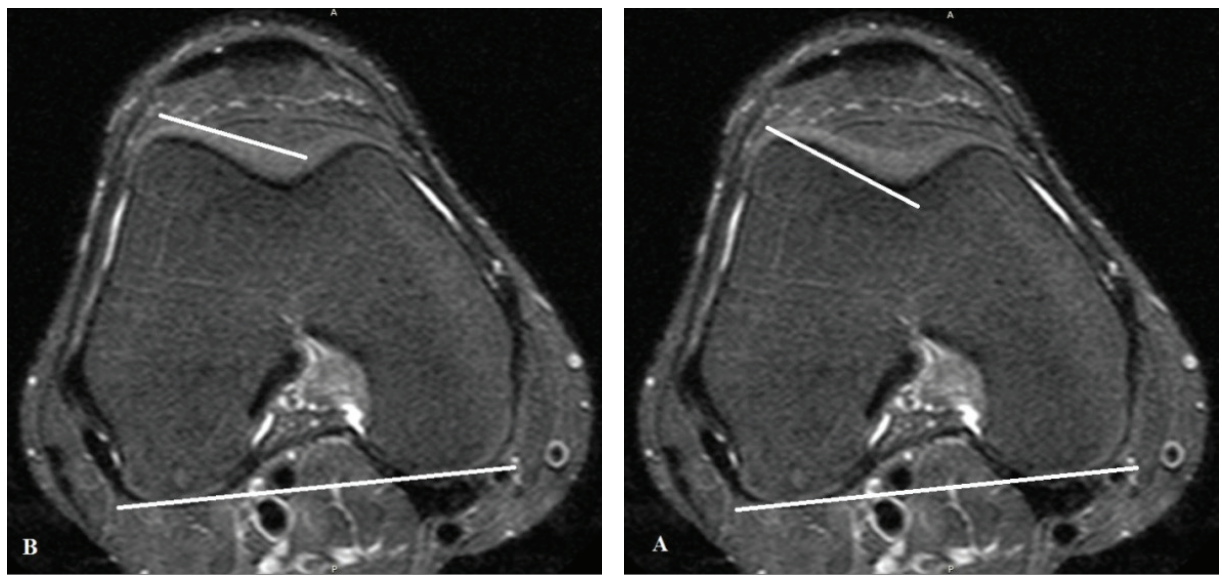

Figure 4. (A) Lateral trochlear inclination angle at bone (LTIB) level is the angle of the lateral ridge of the trochlear groove bone with respect to the posterior condyles, (B) lateral trochlear inclination angle at cartilage level (LTIC) is the angle of the lateral ridge of the trochlear groove cartilage with respect to the posterior condyles

Statistical analysis was performed using SPSS version 20 for Windows (SPSS Inc., Chicago, IL). Student's t-test for independent samples was used to compare means between PFP and pain free groups at all angles of KMRI. Multiple logistic regression was used to determine the effect of trochlea geometry and patella alignment MRI variables on patellofemoral pain. Level of significance was set at $p<0.05$.

\section{Results}

Seventy seven females participated in this study. Fifty one had a diagnosis of PFP (mean \pm standard deviation; age: $25.84 \pm 5.04$ years), twenty six subjects were asymptomatic (mean \pm standard deviation; age: 26.35 \pm 4.77 years).

The descriptive statistics of the trochlea geometry, patellar height in PFP and control groups are presented in Table 1.

Tahle 1. Comparing the trochlea geometry and patellar height indices in PFP and control groups, mean \pm standard deviation

\begin{tabular}{lccc} 
& Pain-free & PFP & $p$ \\
\hline SAB & $135.9 \pm 8.2$ & $143.0 \pm 12.6$ & 0.004 \\
SAC & $146.4 \pm 9.2$ & $153.1 \pm 10.9$ & 0.009 \\
LTIB & $21.3 \pm 4.1$ & $17.4 \pm 6.9$ & 0.003 \\
LTIC & $17.4 \pm 4.2$ & $13.9 \pm 5.9$ & 0.004 \\
ISR & $1.1 \pm 0.2$ & $1.3 \pm 0.2$ & $<0.0001$ \\
\hline
\end{tabular}

SAB: sulcus angle bone; SAC: sulcus angle cartilage; LTIB: lateral trochlear inclination bone; LTIC: lateral trochlear inclination cartilage; ISR: Insall-Salvati ratio.

The means, standard deviations of the variables describing patellar alignment and femoral rotation measurements for both groups are presented in Table 2. 
Table 2. Comparing the patellar alignment and femoral rotation indices in PFP and control groups, mean \pm standard deviation

\begin{tabular}{llcccccc}
\cline { 3 - 7 } & & \multicolumn{5}{c}{ Knee flexion angle } \\
\cline { 3 - 8 } & & $0^{\circ}$ & $10^{\circ}$ & $20^{\circ}$ & $30^{\circ}$ & $40^{\circ}$ & $50^{\circ}$ \\
\hline \multirow{2}{*}{ BSO } & pain-free & $57.2 \pm 4.8^{*}$ & $55.4 \pm 5.2^{*}$ & $53.8 \pm 5.2^{*}$ & $52.3 \pm 4.7^{*}$ & $51.3 \pm 4.8^{*}$ & $50.2 \pm 4.9^{*}$ \\
& PFP & $70.1 \pm 11.7^{*}$ & $65.5 \pm 9.8^{*}$ & $61.2 \pm 7.7^{*}$ & $57.2 \pm 6.1^{*}$ & $55.1 \pm 4.7^{*}$ & $53.9 \pm 4.4^{*}$ \\
\hline \multirow{2}{*}{ PTA } & pain-free & $10.1 \pm 2.7^{*}$ & $8.5 \pm 2.4^{*}$ & $7.5 \pm 2.6^{*}$ & $6.6 \pm 2.6^{*}$ & $5.3 \pm 2.8^{*}$ & $4.1 \pm 3.1^{*}$ \\
& PFP & $13.5 \pm 4.3^{*}$ & $11.8 \pm 4.3^{*}$ & $10.4 \pm 4.2^{*}$ & $9.0 \pm 4.3^{*}$ & $8.2 \pm 3.9^{*}$ & $6.9 \pm 3.9^{*}$ \\
\hline \multirow{2}{*}{ PRA } & pain-free & $4.8 \pm 5.7^{*}$ & $3.8 \pm 5.6^{*}$ & $2.4 \pm 5.6^{*}$ & $1.2 \pm 5.7^{*}$ & $-0.2 \pm 5.3^{*}$ & $-1.2 \pm 5.1^{*}$ \\
& PFP & $0.5 \pm 6.6^{*}$ & $-0.6 \pm 6.7^{*}$ & $-1.6 \pm 6.5^{*}$ & $-2.9 \pm 6.1^{*}$ & $-4.2 \pm 5.3^{*}$ & $-5.0 \pm 4.9^{*}$ \\
\hline \multirow{2}{*}{ FRA } & pain-free & $10.2 \pm 2.6^{*}$ & $7.0 \pm 2.6^{*}$ & $4.9 \pm 3.1^{*}$ & $3.2 \pm 3.3^{*}$ & $1.7 \pm 3.9^{*}$ & $0.7 \pm 4.0^{*}$ \\
& PFP & $18.1 \pm 3.7^{*}$ & $13.2 \pm 3.7^{*}$ & $10.7 \pm 3.8^{*}$ & $8.7 \pm 3.7^{*}$ & $7.3 \pm 3.4^{*}$ & $5.8 \pm 3.0^{*}$ \\
\hline
\end{tabular}

BSO: bisect offset; PTA: patellar tilt angle; PRA: patellar rotation angle; FRA: femoral rotation angle, PFP: patellofemoral pain; ${ }^{*} p<0.05$ comparing pain-free and PFP groups.

As shown in Table $1 \mathrm{SAB}$ was by $7.1^{\circ}$ and SAC was by $6.7^{\circ}$ higher, while LTIB and LTIC were significantly lower in women with PFP compared to healthy women. ISR in females with PFP was 1.3 and it was by 0.2 (18\%) higher than in controls.

In the PFP group compared to the pain-free group, BSO and PTA were higher at all flexion angles $(p<0.01)$. Females with PFP demonstrated greater lateral patellar rotation and greater medial femoral rotation at all flexion angles: PTA was significantly lower in PFP group compared to controls $(p<0.01)$, while FRA was significantly higher in PFP group compared to controls $(p<0.001)$.

A multiple logistic regression (backward based on Wald statistic) analysis was performed on PFP status as outcome and 7 predictors: SAB, LTIB, IS, BSO, PTA, PRA and FRA. Model Nagelkerke $\mathrm{R}^{2}$ was 0.88 . The Table 3 shows regression coefficients, Wald statistic, odds ratio and 95\% confidence intervals for odds ratios.

According to Wald criterion SAB, FRA and PRA reliably predicted PFP status.

Table 3. Odds ratios of factors predicting PFP derived from multiple logistic regression analysis

\begin{tabular}{lcccc}
\hline Variable & Coefficient $(\mathrm{B})$ & $\mathrm{p}$ & Odds ratio & $95 \% \mathrm{Cl}$ for odds ratio \\
\hline SAB & 0.5 & 0.039 & 1.6 & $1.0-2.6$ \\
LTIB & 0.7 & 0.077 & 2.0 & $0.9-4.6$ \\
ISR & -15.2 & 0.060 & 0.0 & $0.0-1.9$ \\
FRA & 1.7 & 0.005 & 5.6 & $1.7-18.6$ \\
PRA & -0.5 & 0.008 & 0.6 & $0.4-0.9$ \\
\hline
\end{tabular}

SAB: sulcus angle bone; LTIB: lateral trochlear inclination bone; ISR: Insall-Salvati ratio; FRA: femoral rotation angle, PRA: patellar rotation angle.

Odds analysis demonstrated that increase of FRA by $1^{\circ}$ increased the likelihood of PFP 5.6 times, increase of SAB by $1^{\circ}$ increased the likelihood of PFP by 1.6 times. Decrease of PRA by $1^{\circ}$ decrease PFP likelihood by 1.7 times. 


\section{Discussion}

In our study females with PFP had shallower femoral sulcus at subchondral bone level as well at cartilage level compared to the healthy volunteers. Majority of investigations demonstrated that increase in the sulcus angle is indicative of compromised patellar stability and PFP (Powers 2000; Varadarajan et al. 2010). Our findings were consistent with Powers et al. (2000) results who reported a substantial loss of the bony groove depth in subjects with PFP as the knee extended beyond $27^{\circ}$ with approximately $9^{\circ}$ higher SAB at full extension in subjects with PFP compared to healthy volunteers. In our study subjects' with PFP SAB was by $7.1^{\circ}$ higher and SAC was by $6.7^{\circ}$ higher compared to the controls.

Teng et al. (2014) reported higher SAB values in asymptomatic controls and in women with PFP compared to our results, as well as smaller difference between healthy and in females with PFP. Balcarek et al. (2010) reported $154.6 \pm 11.6^{\circ} \mathrm{SAB}$ in patients with patellar dislocation, which was by $8.4^{\circ}$ higher than in healthy women. Examination of 566 knees ( $59 \%$ of them were female) in patients who either had knee osteoarthritis or were at high risk (older than 50 years, overweight, or had a previous knee injury) for knee osteoarthritis revealed $S A B$ at $130.4^{\circ} \pm 8.7^{\circ}$ (Stefanik et al. 2012). Our results showed lower SAB than reported by Balcarek et al. (2010) because his data was collected from patients treated for lateral patellar instability, which is associated with shallower sulcus. On the contrary, data presented by Stefanik et al. (2012) showed deeper sulcus for patellofemoral osteoarthritic patients, because they were selected with risk factors that predispose osteoarthritis despite sulcus depth.

In our study females with PFP had higher lateral trochlea inclination at subchondral bone level as well at cartilage level compared to the healthy volunteers. Carrillon et al. (2000) were the first to describe the use of the lateral trochlear inclination on MRI. They reported mean values of $6.2^{\circ}$ in subjects with a history of patella dislocation and $16.9^{\circ}$ in control subjects $\left(p<0.001\right.$ ). Teng et al. (2014) reported mean LTIB values of $14.9^{\circ} \pm 5.4^{\circ}$ in women with PFP and $17.9^{\circ} \pm 8.3^{\circ}$ in asymptomatic controls. In our study LTIB in women with PFP was $17.4 \pm 6.9^{\circ}$ and in controls $21.3 \pm 4.1^{\circ}$. Our results are consistent with the results of other investigators in that a lower lateral trochlear inclination angle (i.e., flattened lateral trochlea) was associated with the compromised patellar stability and PFP.

Our study demonstrated greater patella height in PFP subjects compared to pain free subjects using ISR. Our results were consistent with Pal et al., (2013) who reported greater patella height in chondromalacia patella subjects compared to pain free subjects using the ISR. Pal et al. (2013) reported that average patella height for PFP subjects was even $30 \%$ greater than the pain free subjects using the Insall-Salvati index $(p<0.001)$.

Based on values of BSO, patellar lateralization was confirmed in both groups at all angles of KMRI. Despite it, the subjects with PFP demonstrated greater statistically significant patellar lateralization at all angles of flexion compared to healthy women. This finding coincide with results of Souza et al. (2010). The largest difference between groups was evident at full extension ( $70.1 \%$ versus $57.2 \%$ of the patella lateral to the midline), which was consistent with Powers (2000) results and coincided with the contention of Fulkerson (2002) that patellar subluxation typically occurs during terminal knee extension.

The patellar tilt data showed that the patella was laterally tilted throughout the range of motion in both groups. The subjects with PFP demonstrated a greater magnitude compared with the subjects without PFP at all knee flexion angles with the largest difference between groups was evident at full extension. The subjects with and without PFP demonstrated an overall pattern of increasing lateral tilt as the knee extended, which was consistent with other authors (Biyani et al. 2014; Teng et al. 2014). 
Patella rotation significantly differed between groups. The progressive internal rotation of the patella with knee extension was observed in both groups, but in women with PFP group internal patellar rotation was evident from $10^{\circ}$ of knee flexion angle while in control group internal patella rotation occurred at $40^{\circ}$ and $50^{\circ}$ of knee flexion. Our results are in agreement to data of Souza et al. (2010). They reported about slight lateral rotation of patella across all knee flexion angles and there was a tendency for the patella to rotate medially as the knee extended in PFP and control groups. For pain free group patellar rotation demonstrated grater positive values (i.e. greater medial rotation). Differences might have occurred because of different patient positioning. We used supine, while Souza et al. (2010) used upright scanning position. The pattern of motion we observed is in contrast to what has been reported by Powers et al. (2000) during non-weight-bearing conditions.

Women with PFP demonstrated greater medial femoral rotation compared to the control group at all knee flexion angles. The largest difference between groups was observed at $0^{\circ}$ of knee flexion, where the subjects with PFP had $180 \%$ of the amount of medial femoral rotation compared to the control group $\left(10.2^{\circ} \pm 2.6^{\circ}\right.$ vs. $\left.18.1^{\circ} \pm 3.7^{\circ}\right)$. The maximum medial rotation exhibited in our PFP group $\left(18.1^{\circ}\right)$ was higher than previously reported by Powers et al. $\left(13^{\circ}\right)$ and Souza et al. $\left(12.2^{\circ}\right)$, who used a similar weight-bearing protocol to that employed by the current study. Greater medial femoral rotation may be predisposed by skeletal abnormalities such as femoral anteversion or hip muscle weakness, which was not evaluated in the present study. Another possible reason for greater medial femoral rotation could be "screw-home mechanism", which occurs at end stage of extension and knee joint locking (Wittstein et al. 2009; Dietrich et al. 2014; Izadpanah et al. 2014).

\section{Conclusions}

This kinematic magnetic resonance imaging study revealed femoral rotation angle, sulcus angle at bone level and patellar rotation angle to have best predictive value for patellofemoral pain. Presented results can help to assess the complexity of predisposing factors for PFP in practice.

\section{References}

Balcarek P., Jung K., Ammon J., Walde T.A., Frosch S., Schüttrumpf J.P., Stürmer K.M., Frosch K.H. Anatomy of lateral patellar instability: trochlear dysplasia and tibial tubercle-trochlear groove distance is more pronounced in women who dislocate the patella. Am J Sports Med. 2010; 38: 2320-2327.

Biyani R., Elias J.J., Saranathan A., Feng H., Guseila L.M., Morscher M.A., Jones K.C. Anatomical factors influencing patellar tracking in the unstable patellofemoral joint. Knee Surg Sports Traumatol Arthrosc 2014; 22: 2334-2341.

Brossmann J., Muhle C., Schroder C., Melchert U.H., Bull C.C., Spielmann R.P., Heller M. Patellar tracking patterns during active and passive knee extension: evaluation with motion-triggered cine MR imaging. Radiology 1993; 187: 205-212.

Carrillon Y., Abidi H., Dejour D., Fantino O., Moyen B., Tran-Minh V.A. Patellar instability: assessment on MR images by measuring the lateral trochlear inclination-initial experience. Radiology 2000; 216: 582-585.

Dietrich T.J., Betz M., Pfirrmann C.W., Koch P.P., Fucentese S.F. End-stage extension of the knee and its influence on tibial tuberositytrochlear groove distance (TTTG) in asymptomatic volunteers. Knee Surg Sports Traumatol Arthrosc. 2014; 22: $214-218$.

Fulkerson J.P. Diagnosis and treatment of patients with patellofemoral pain. Am J Sports Med. 2002; 30 (3): 447-456.

Harbaugh C.M., Wilson N.A., Sheehan F.T. Correlating femoral shape with patellar kinematics in patients with patellofemoral pain. J Orthop Res. 2010; 28: 865-872.

Insall J.N., Aglietti P., Tria Jr A.J. Patellar pain and incongruence. II: Clinical application. Clin Orthop Relat Res.1983; 176: 225-232.

Izadpanah K., Weitzel E., Vicari M., Hennig J., Weigel M., Südkamp N.P., Niemeyer P. Influence of knee flexion angle and weight bearing on the Tibial Tuberosity-Trochlear Groove (TTTG) distance for evaluation of patellofemoral alignment. Knee Surg Sports Traumatol Arthrosc. 2014; 22: 2655-2661. 
Pal S., Besier T.F., Beaupre G.S., Fredericson M., Delp S.L., Gold G.E. Patellar maltracking is prevalent among patellofemoral pain subjects with patella alta: an upright, weightbearing MRI study. J Orthop Res. 2013; 31: 448-457.

Powers C.M. Patellar kinematics, part II: the influence of the depth of the trochlear groove in subjects with and without patellofemoral pain. Phys Ther. 2000; 80: 965-978.

Powers C.M. The influence of abnormal hip mechanics on knee injury: a biomechanical perspective. J Orthop Sports Phys Ther. 2010; 40: 42-51.

Shih Y.F., Bull A.M., Amis A. The cartilaginous and osseous geometry of the femoral trochlear groove. Knee Surg Sports Traumatol Arthrosc. 2004; 12: 300-306.

Souza R.B., Draper C.E., Fredericson M., Powers C.M. Femur rotation and patellofemoral joint kinematics: a weight-bearing magnetic resonance imaging analysis. J Orthop Sports Phys Ther. 2010; 40: 277-285.

Staubli H.U., Durrenmatt U., Porcellini B. Rauschning W. Anatomy and surface geometry of the patellofemoral joint in the axial plane. J Bone Joint Surg Br. 1999; 81: 452-458.

Stefanik J.J., Roemer F.W., Zumwalt A.C., Zhu Y., Gross K.D., Lynch J.A., Frey-Law L.A., Lewis C.E., Guermazi A., Powers C.M., Felson D.T. Association between measures of trochlear morphology and structural features of patellofemoral joint osteoarthritis on MRI: the MOST study. J Orthop Res. 2012; 30: 1-8.

Teng H.L., Chen Y.J., Powers C.M. Predictors of patellar alignment during weight bearing: an examination of patellar height and trochlear geometry. Knee. 2014; 21: 142-146.

Varadarajan K.M., Freiberg A.A., Gill T.J., Rubash H.E., Li G. Relationship between three-dimensional geometry of the trochlear groove and in vivo patellar tracking during weight-bearing knee flexion. J Biomech Eng. 2010; 132: 061008.

Ward S.R., Terk M.R., Powers C.M. Patella alta: association with patellofemoral alignment and changes in contact area during weightbearing. J Bone Joint Surg Am. 2007; 89: 1749-1755.

Wilson T. The measurement of patellar alignment in patellofemoral pain syndrome: are we confusing assumptions with evidence? J Orthop Sports Phys Ther. 2007; 37: 330-341.

Wittstein J.R., Bartlett E.C., Easterbrook J., Byrd J.C. Magnetic resonance imaging evaluation of patellofemoral malalignment. Arthroscopy 2006; 22: 643-649.

Wittstein J.R., O'Brien S.D., Vinson E.N., Garrett, Garrett, Jr W.E. MRI evaluation of anterior knee pain: predicting response to nonoperative treatment. Skeletal Radiol. 2009; 38: 895-901.

Witvrouw E., Callaghan M.J., Stefanik J.J., Noehren B., Bazett-Jones D.M., Willson J.D., Earl-Boehm J.E., Davis I.S., Powers C.M., McConnell J., Crossley K.M. Patellofemoral pain: consensus statement from the 3rd International Patellofemoral Pain Research Retreat held in Vancouver, September 2013. Br J Sports Med. 2014; 48: 411-414.

Cite this anticle aS: Brazaitis A., Tamošiūnas A., Tutkuvienè J., Gocentas A. Predictors of Patellofemoral Pain Applying Full Weight Bearing Kinematic MRI. Central European Journal of Sport Sciences and Medicine. 2015; 12 (4): 43-51. 
